\title{
Ultra cool dwarfs with companions
}

\author{
R. G. Kurtev ${ }^{1}$, V. D. Ivanov ${ }^{2}$, R. Jayawardhana ${ }^{3}$ \\ and J. H. Borissova ${ }^{1}$ \\ ${ }^{1}$ Department of Physics and Astronomy, Valparaiso University, Chile \\ email: radostin.kurtev@uv.cl; jura.borissova@uv.cl \\ ${ }^{2}$ European Southern Observatory, Santiago, Chile, email: vivanov@eso.org \\ 3 Toronto University, Canada, email: rayjay@astro.utoronto.ca
}

The binary fraction in the sub-stellar regime is a topic of discussion. The lower masses of ultra cool dwarfs (UCDs) with respect to the other stars make them even more important because a measurable effect on their radial velocity (RV) or luminosities can be caused by extremely low mass companions. Some UCDs in young star forming regions are bright enough to be studied with existing high resolution instrumentation. The UCDs are intrinsically faint in the optical and the optical RV measurements are affected by "rotationally modulated inhomogeneous surface features" that can mimic a companion, while the near-infrared (NIR) RVs are less prone to them. Therefore, we decided to monitor the RV of six UCDs in the NIR. Blake et al. (2007) demonstrated RV measurement accuracy of $300-600 \mathrm{~m} \mathrm{~s}^{-1}$ in the NIR using telluric calibration.

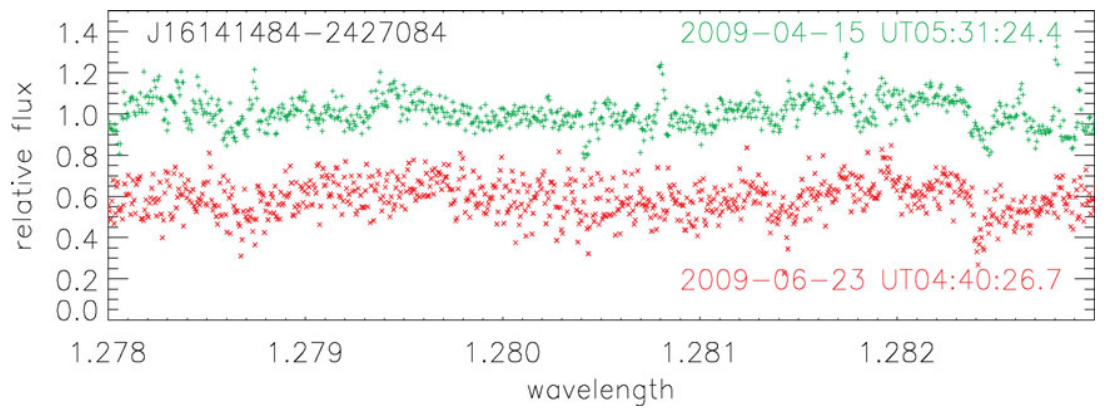

Figure 1. Spectra of J16141484-2427084 obtained at two epochs.

Here we present the first results of a search for unresolved companions (with NIR measured RV) around a sample of young low mass members of the Upper Sco star-forming region based on high resolution (R 50000) $J$-band GEMINI(S)/PHOENIX spectra obtained during the program GS-2009A-Q-68. The spectra for two epochs of J16141484-2427084 are presented in Fig 1. Our targets were selected from the list of Slesnick et al. (2006). Two of them, J16141484-2427084 and J16095217-2136283, present radial velocity variations above the sensitivity threshold of about $700-900 \mathrm{~m} \mathrm{~s}^{-1}$. The obtained $\Delta V$, corrected for the Earth barycentric velocity, are $3.58 \pm 0.73$ $\mathrm{km} \mathrm{s}^{-1}$ and $-2.61 \pm 0.93 \mathrm{~km} \mathrm{~s}^{-1}$ respectively. A further model analysis will be carried out to improve the precision and to search for differences in the radial velocity of other candidates.

RGK and JHB would like to acknowledge the support from FONDECYT projects \#1080154 and \#1080086.

\section{References}

Blake et al. 2007, ApJ, 666, 1198

Slesnick et al. 2006, AJ, 131, 3016 